\title{
Possibility of combining carbon dots and liquid exfoliated graphene as a carbon based light addressable potentiometric sensor
}

Fang Li, Jizhao Zhang, Shihui Hu, Yunfang Jia*

College of Electronic Information and Optical Engineering, Nankai University, Tianjin, China.

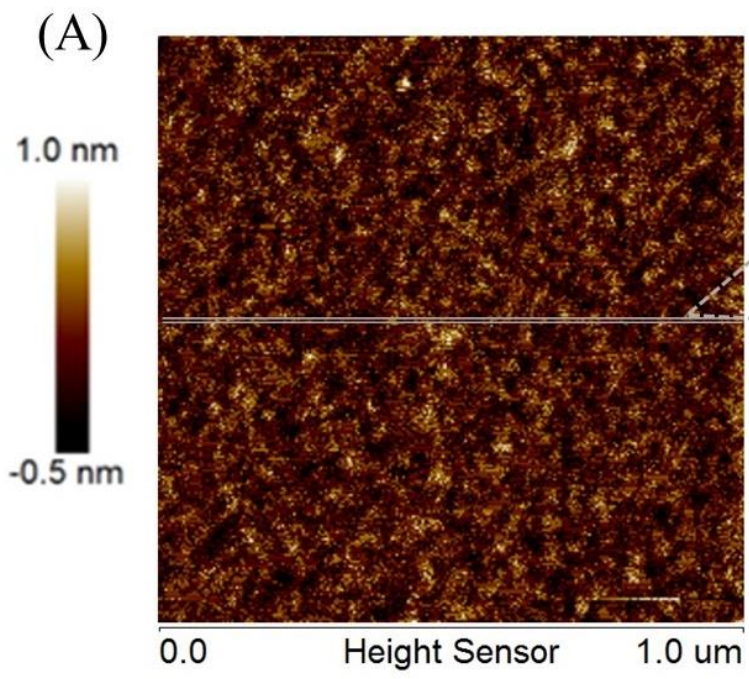

(B)

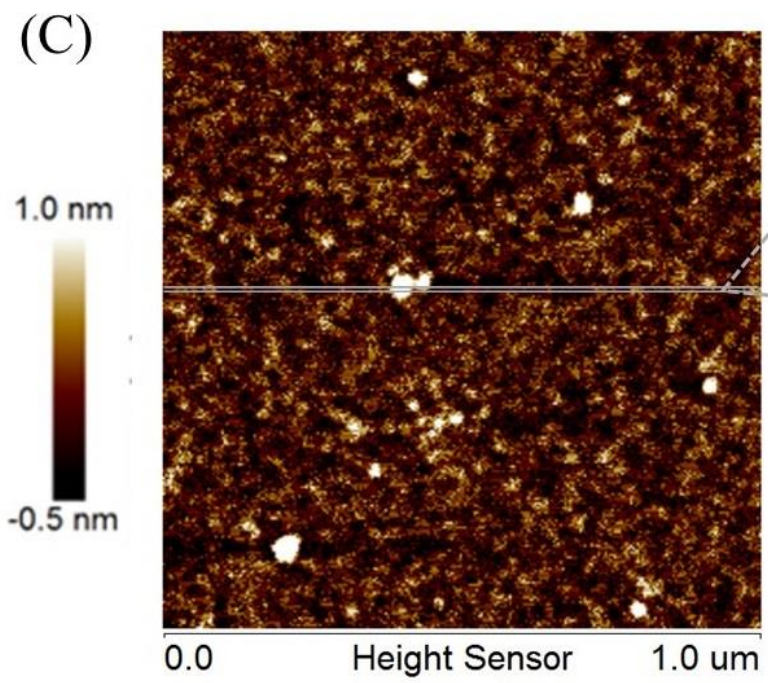

(D)

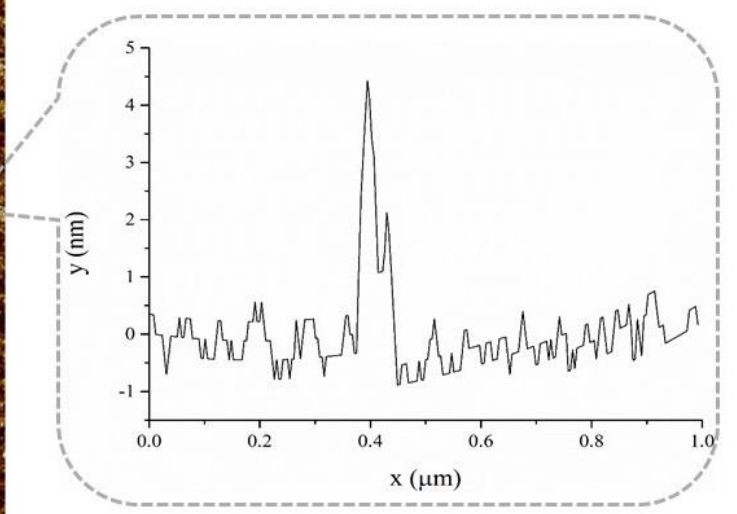

Figure S1. AFM characterizations of blank silicon wafer (A), carbon-dots (CDs) decorated silicon wafer (C). Their height distributions are analyzed by using NanoScope analysis software, one of the height distributions along the gray line are shown in (B) and (D), respectively. According to the height analyses, the thickness of CDs layer is about $2-4 \mathrm{~nm}$. 

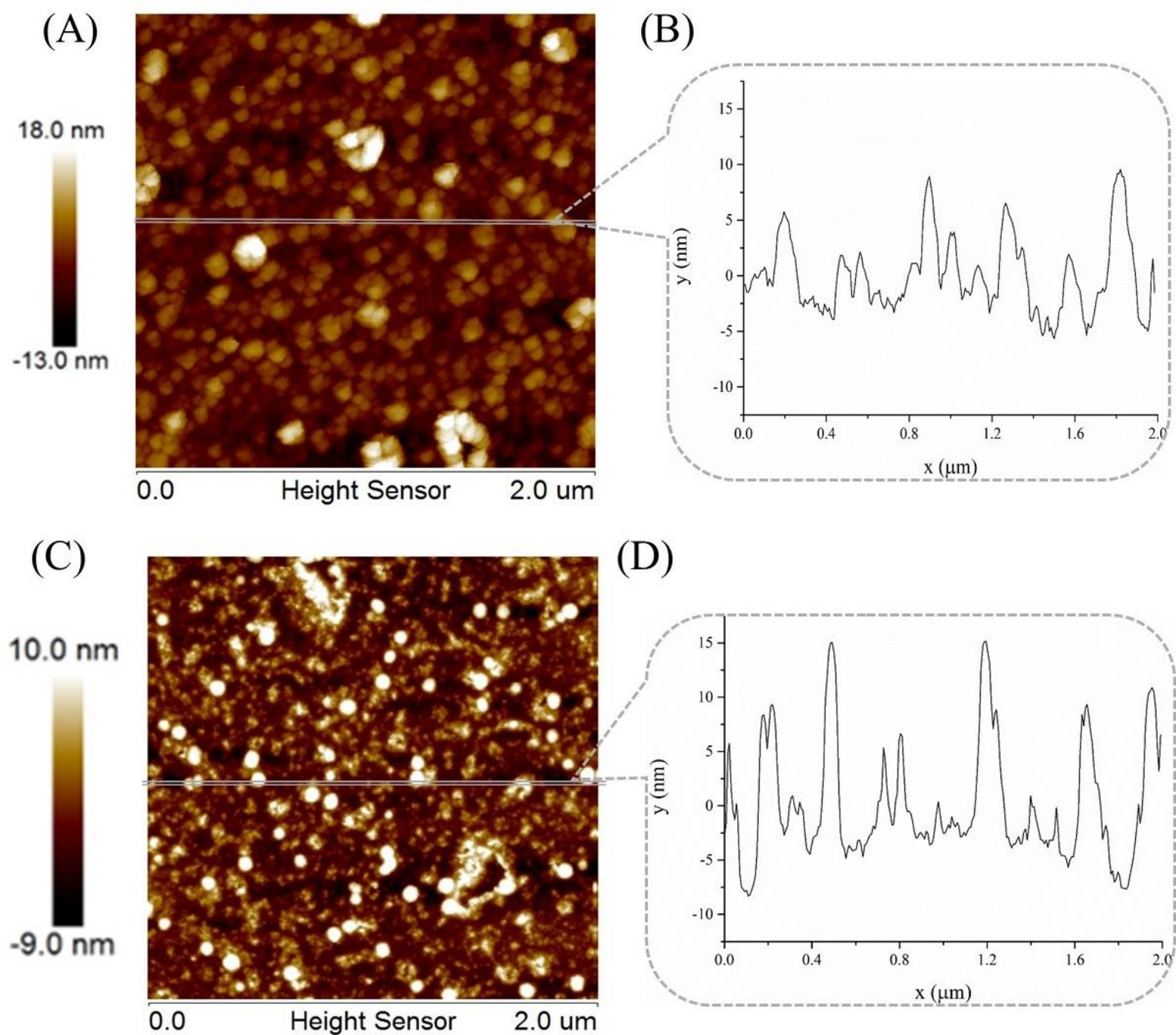

(D)

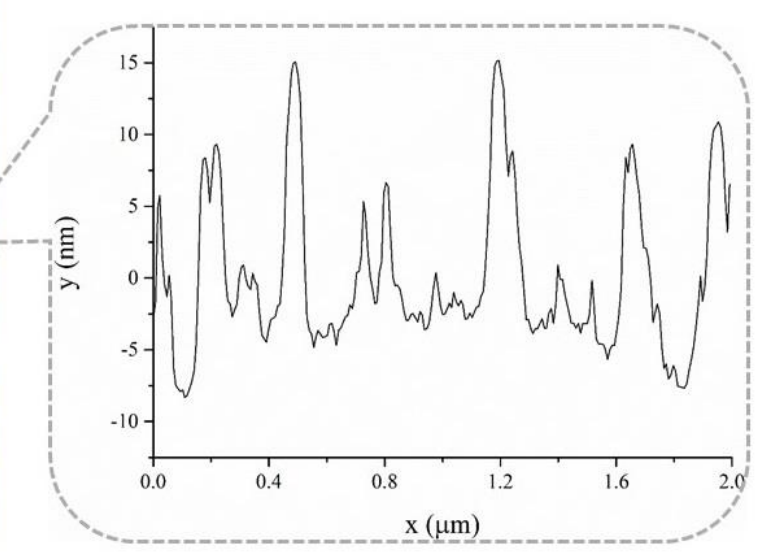

Figure S2. AFM characterizations of the cleaned glass slide (A), carbon-dots (CDs) decorated slide (B). Their height distributions are analyzed by using NanoScope analysis software, one of the height distributions along the gray line are shown in (C) and (D), respectively. According to the height analyses, the thickness of CDs layer is about $5 \mathrm{~nm}$.
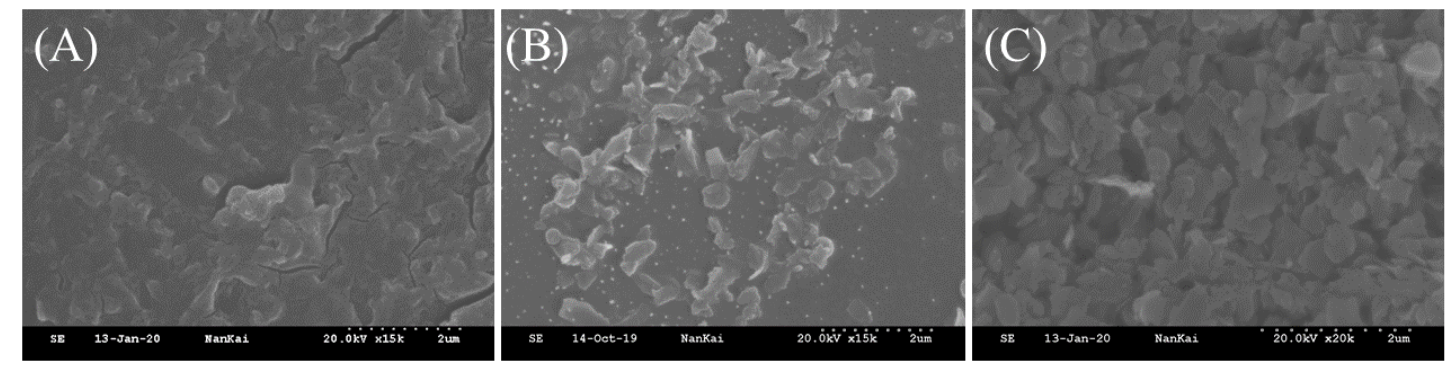

Figure S3. SEM photographs of (A) liquid exfoliated grahene (LEG) coated on slide, (B) the LEG coated on CDs decorated slide, (C) the CDs coated on LEG decorated slide. It is found the nano-sheets of LEG are overlapped in (A), meanwhile, the edges of LEG and their wrinkles in them are clear. In (B), the CDs are identified by the small bright points, and LEG nano-sheets tend to be in an erect form. In (C), there are stacked LEG nanoflakes which are similar to the nanosheets in (A), but there is the difference between them, the edges of LEG flake in (C) are fuzzy than in (A). The fluffy surface of the CDs/LEG sample (Figure S3C), in comparison with LEG (Figure S3A) and LEG/CDs (Figure S3B) indicates more CDs are deposited. 

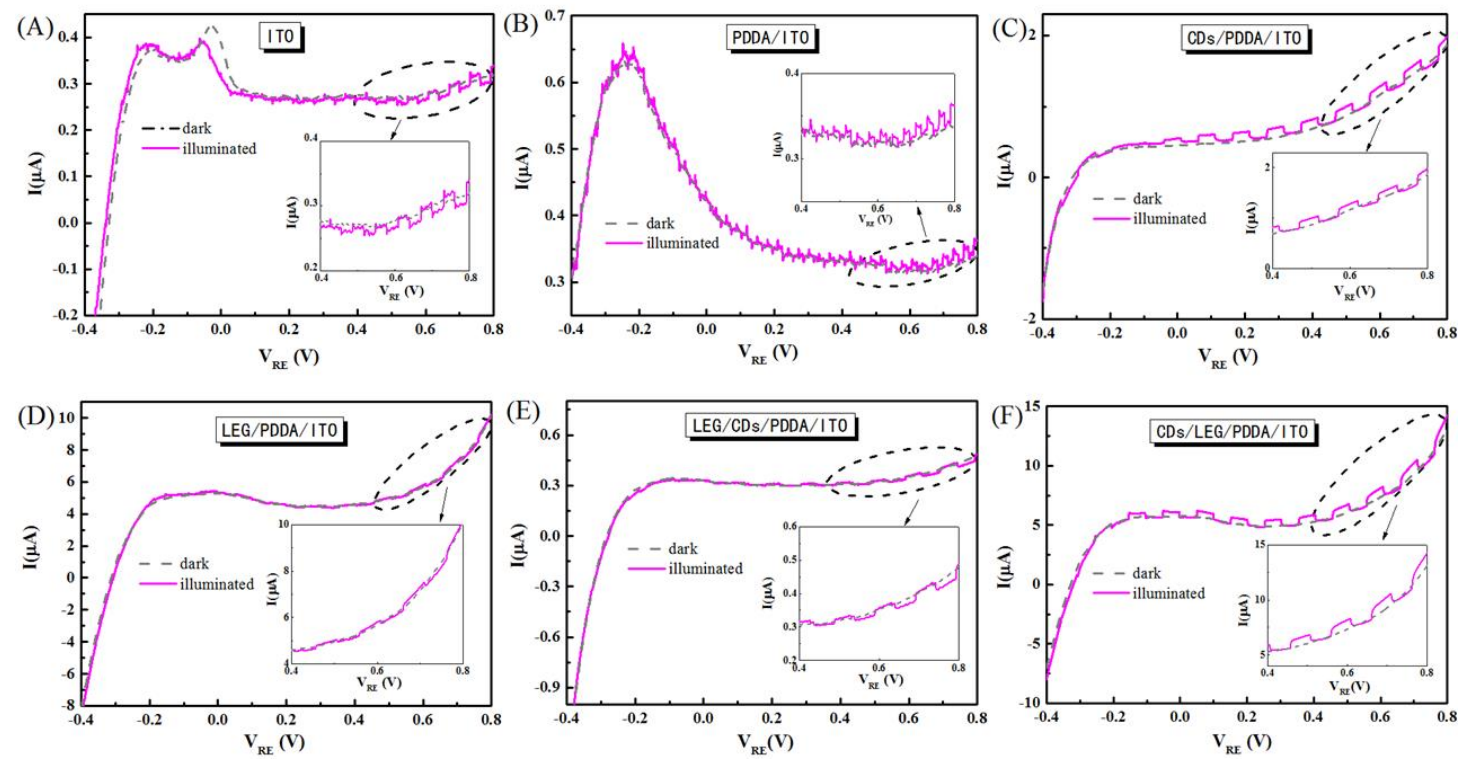

Figure S4. The LSV curves of (A)ITO, (B) PDDA decorated ITO substrates (PDDA/ITO), (C) assembling CDs on PDDA/ITO (CDs@PDDA/ITO), (D) assembling LEG on PDDA/ITO (LEG@PDDA/ITO), (E) drop-coating LEG on CDs@PDDA/ITO (LEG/CDs@PDDA/ITO), (F) drop-coating CDs on LEG@PDDA/ITO (CDs/LEG@PDDA/ITO) under the conditions of dark, illumination and chopped illumination with scan rates of $30 \mathrm{mV} \mathrm{s}^{-1}$.

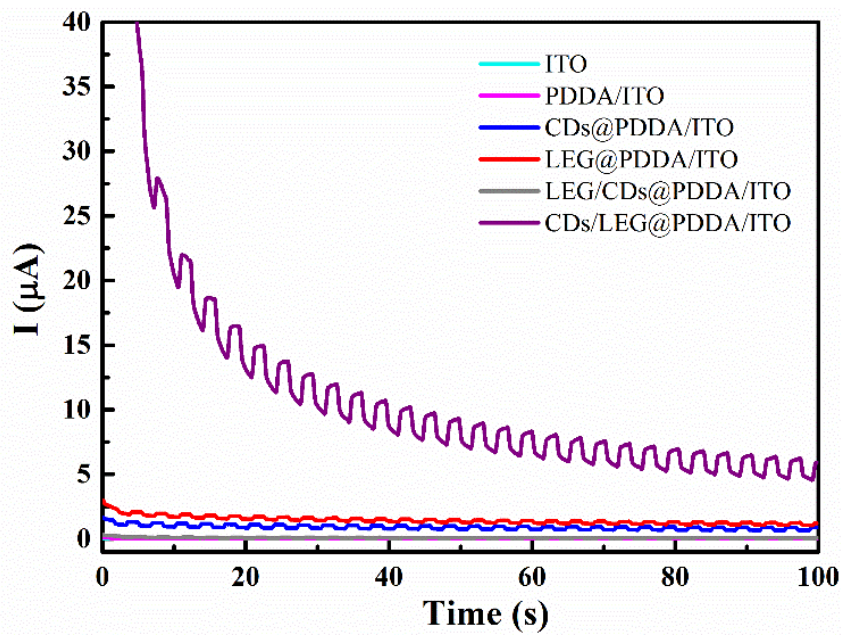

Figure S5. The transient current curves of ITO, PDDA/ITO, CDs@PDDA/ITO, LEG@PDDA/ITO, LEG/CDs@PDDA/ITO and CDs/LEG@PDDA/ITO at $0.8 \mathrm{~V}$, under the chopped illumination. It can be seen that more time is needed for CDs/LEG@PDDA/ITO to reach the stable photo-electronic responding. The conjecture for this phenomenon is based on the theoretical analyses in manuscript (the section of "Working mechanism of C-LAPS" and Figure 5E). We deduce the equivalent capacitance $\left(C_{\mathrm{B}}\right)$ and the varied parasitic resistor $\left(R_{\mathrm{p}}\right)$ are the main factors. First, the currents of CDs@PDDA/ITO (blue) and LEG@PDDA/ITO (red) indicate their resistors in "light on" are bigger than "light off". That means the $R_{\mathrm{p}}$ in CDs/LEG@PDDA/ITO is varied under the chopped illumination. Second, according the working mechanisms of C-LAPS in manuscript, the existence of the $C_{\mathrm{B}}$ in CDs/LEG@PDDA/ITO is the main distinction from the other devices. The electric field in it can separate more photo-generated electron-hole pairs than CDs@ PDDA/ITO and LEG@PDDA/ITO, so the highest currents can be understood. However, when the light is off, there will be more unbalanced electrons and holes in the device CDs/LEG@PDDA/ITO to be recombined, so it will take more time $(60 \mathrm{~s})$ to reach the steady-state. 


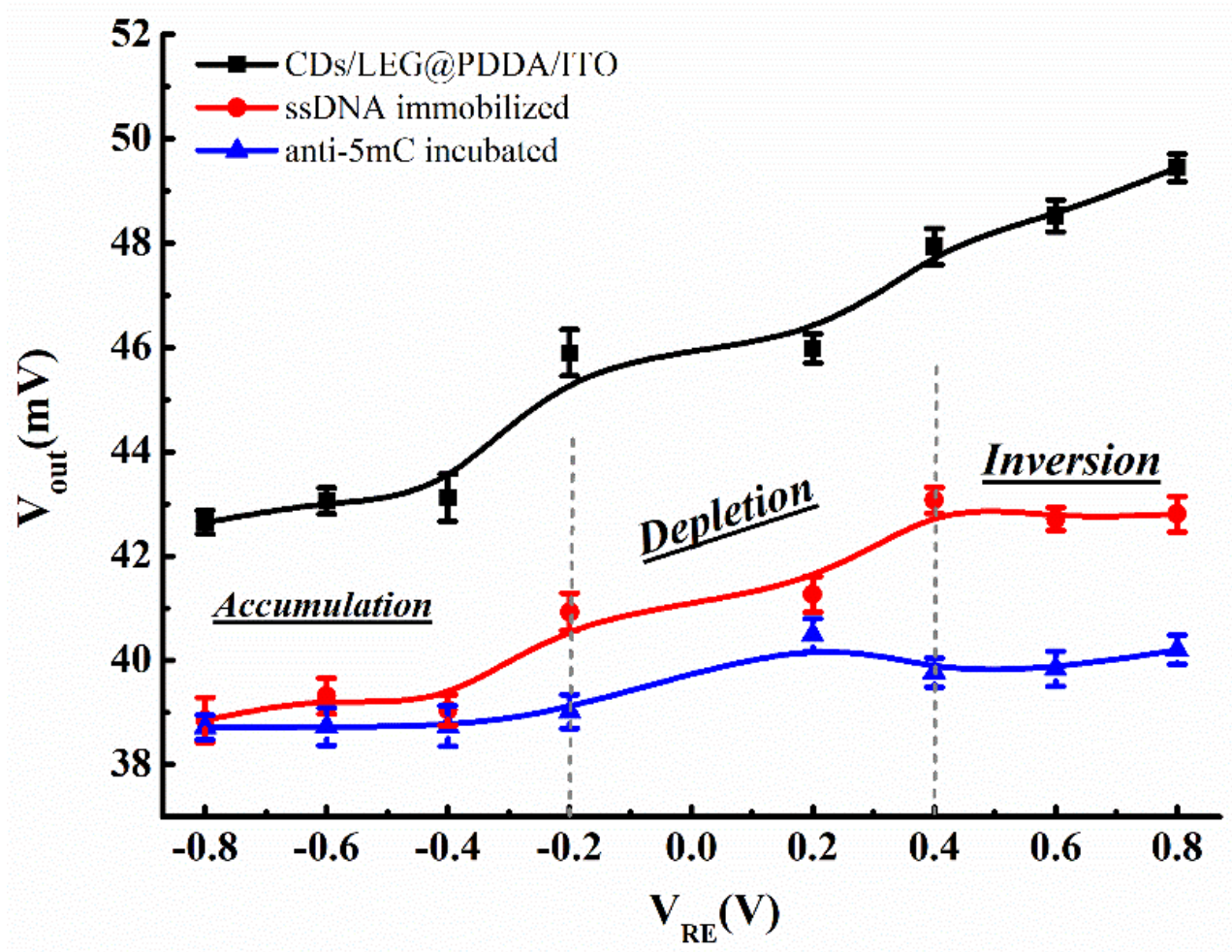

Figure S6. I-V curves of C-LAPS measured by LAPS detection method. The three curves are the concerned C-LAPS with the structure of CDs/LEG@PDDA/ITO, and after they are modified by ssDNA and incubated with anti-5mC solution. The field effect induced three kinds of interfacial states of LAPS can be identified, which are the accumulation, depletion and inversion states.
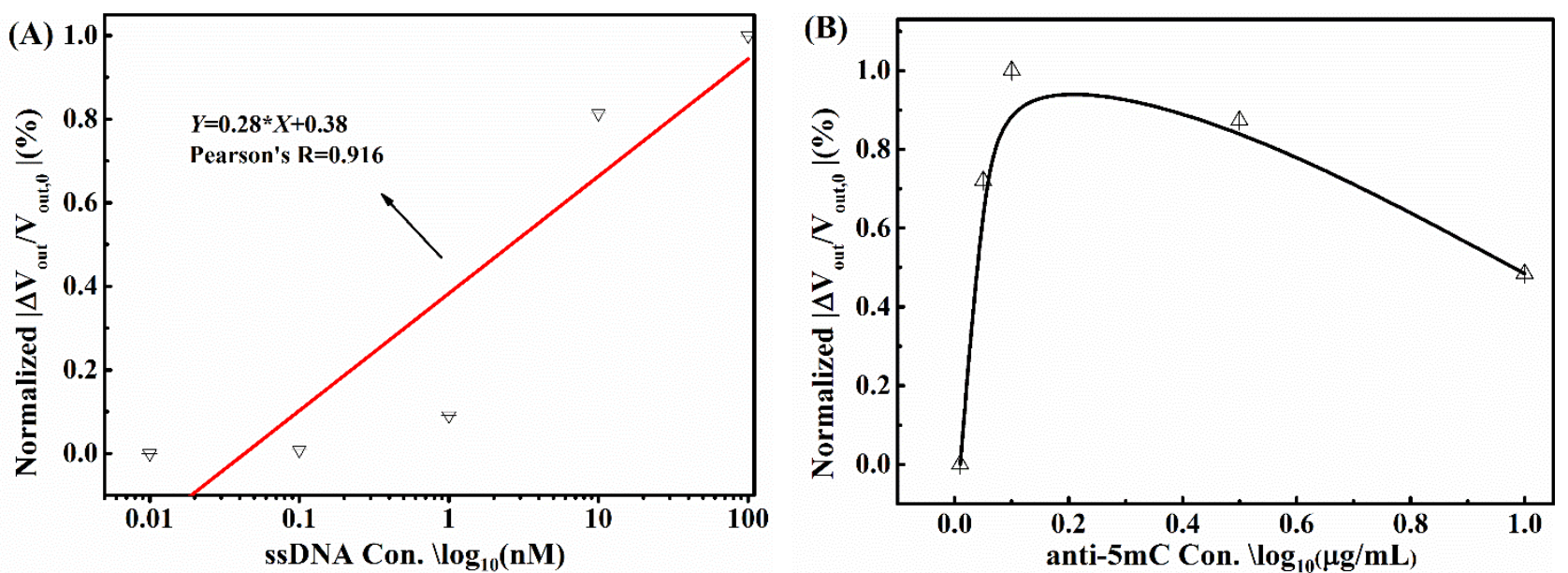

Figure S7. The numerical data of previous Si-LAPS' responding for by ssDNA immobilization (A) and anti-5mC incubations (B). ${ }^{15}$ $\Delta V_{\text {out }}$ in (A) and (B) is defined as $\Delta V_{\text {out }}=V_{\text {out }}-V_{\text {out }, 0}, V_{\text {out }}$ and $V_{\text {out }, 0}$ are Si-LAPS' output voltages before and after being incubated with different concentrations of ssDNA and anti-5mC. 


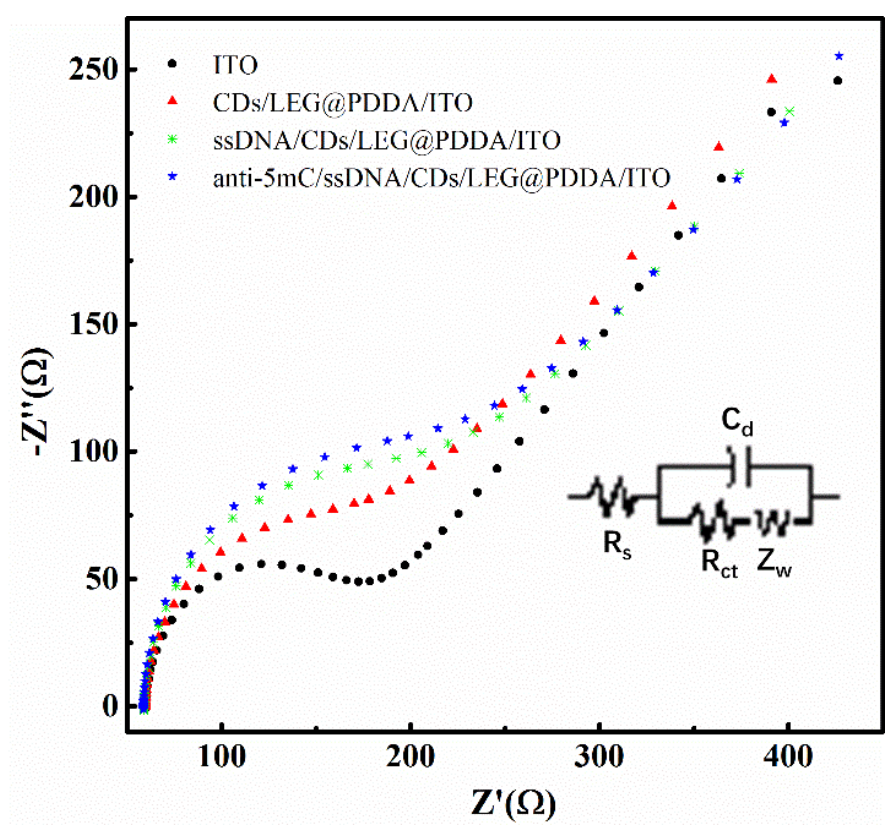

Figure S8. Electrochemical impedance spectra (EIS) of the proposed C-LAPS on ITO substrate, named as CDs/LEG@PDDA/ITO, the ssDNA anchored C-LAPS (ssDNA/CDs/LEG@PDDA/ITO) and after being incubated with anti-5mC (anti5mC/ssDNA/CDs/LEG@PDDA/ITO). Meanwhile, the blank ITO’s EIS is also presented as control. Nyquist plots are used at here. The charge transfer resistances $\left(R_{\mathrm{ct}}\right)$ in Nyquist equivalent circuit are calculated by software Zsimp, which are $101.4 \Omega$ (ITO), $105.8 \Omega$ (CDs/LEG@PDDA/ITO), $136.3 \Omega$ (ssDNA/CDs/LEG@PDDA/ITO) and $145.6 \Omega$ (anti-5mC/ssDNA/CDs/LEG@PDDA/ITO).

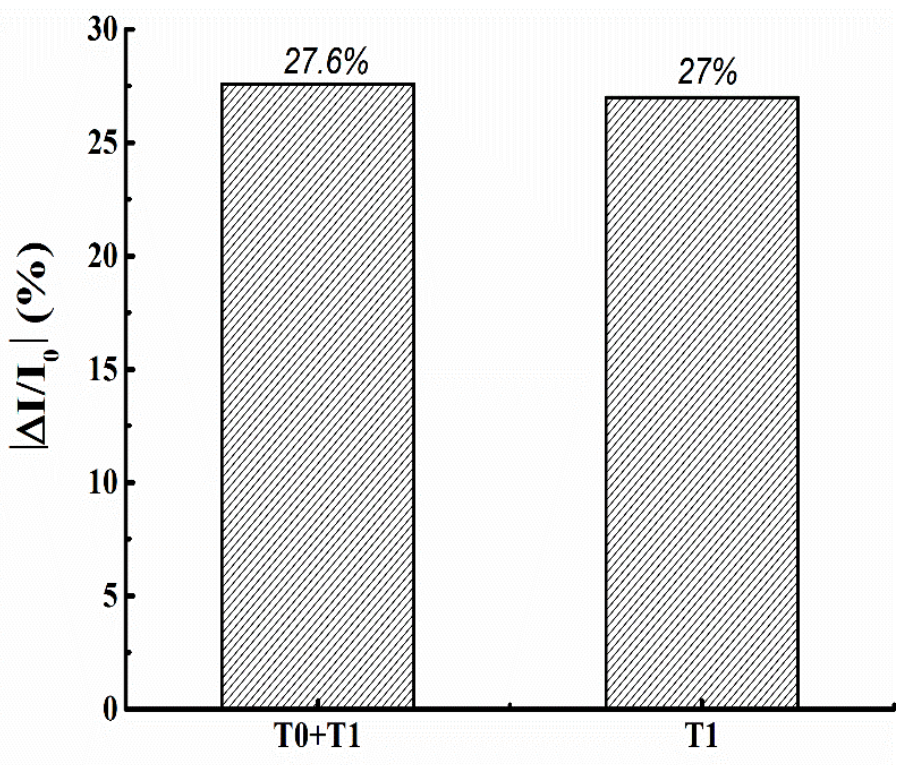

Figure S9. The responses of C-LAPS in the control experiment following the procedure described in the manuscript. T1 is the testing ssDNA solution with the concentration $100 \mathrm{nM}$. T0 is the unmethylated ssDNA with the concentration $100 \mathrm{nM}$. Their mixture is named as T0 $+\mathrm{T} 1$. The current change $\Delta I$ is defined as $\Delta I=I-I_{0}, I_{0}$ and $I$ are the $I_{\mathrm{ph}}$ of C-LAPS with the structure of CDs/LEG@ PDDA/ITO before and after being incubated with anti-5mC. 